\title{
ON ISOMORPHISMS WITH SMALL BOUND
}

\author{
MICHAEL, CAMBERN
}

If $X$ and $Y$ are locally compact Hausdorff spaces, and if we denote by $C_{0}(X)$ and $C_{0}(Y)$ the Banach spaces of continuous, complexvalued functions vanishing at infinity on $X$ and $Y$ respectively, then, according to the Banach-Stone Theorem, the existence of an isometry between the function spaces $C_{0}(X)$ and $C_{0}(Y)$ implies that $X$ and $Y$ are homeomorphic. In [1] it was shown that if we assume, in addition, that $X$ and $Y$ satisfy the first axiom of countability, then this theorem can be generalized by considering isomorphisms with small norm instead of isometries. The object of this paper is to show that the conclusions of [1] remain valid without the assumption of first countability.

Theorem. Let $X$ and $Y$ be locally compact Hausdorff spaces. If there exists a norm-increasing isomorphism $\phi$ of $C_{0}(X)$ onto $C_{0}(Y)$ with bound less than two, $\|f\| \leqq\|\phi(f)\| \leqq\|\phi\|\|f\|, f \in C_{0}(X),\|\phi\|<2$, then $X$ and $Y$ are homeomorphic.

Proof. We employ the notation of [1]. $M$ will denote a real number with $\|\phi\|<2 M<2$, and we define the constants $M^{\prime}, N, N^{\prime}$ by $M^{\prime}=\|\phi\|-M, N=1 / 2 M, N^{\prime}=1-N$. For any point $x \in X$ (resp. $y \in Y$ ) we denote by $\mu_{x}$ (resp. $\mu_{y}$ ) the positive unit mass concentrated at the point $x$ (resp. $y$ ). We then denote by $Y_{1}$ the set of all $y \in Y$ for which there exists an $x \in X$ with

$$
\phi^{*} \mu_{y}=\alpha \mu_{x}+\mu,
$$

where $\alpha$ is a complex number with $|\alpha|>M$, and $\mu$ is a regular Borel measure on $X$ (element of $\left.C_{0}(X)^{*}\right)$ with $\mu(\{x\})=0$, and hence $\|\mu\|<M^{\prime}$. We thus obtain a mapping $\rho$ from $Y_{1}$ to $X$ defined by $\rho(y)=x$, where $x$ is associated with $y$ by (1).

Similarly, we let $X_{1}$ represent the set of all $x \in X$ for which there exists a $y \in Y$ with

$$
\phi^{*-1} \mu_{x}=\beta \mu_{y}+\mu,
$$

where $\beta$ is a complex number with $|\beta|>N$, and $\mu$ is a regular Borel measure on $Y$ with $\mu(\{y\})=0$, and hence $\|\mu\|<N^{\prime}$. A mapping $\tau$ of $X_{1}$ to $Y$ is then defined by $\tau(x)=y$, where $y$ is associated with $x$ by (2).

Received by the editors October 4, 1966. 
What we want to show is that $Y_{1}=Y$, and that $\rho$ is a homeomorphism of $Y$ onto $X$ which has $\tau$ as its inverse. In [1], this was established by a sequence of three propositions. The proofs of the first two of these depended upon an application of the dominated convergence theorem, for which some countability hypothesis was necessary. Here we establish the theorem by proving the same three propositions, but in the absence of a countability condition the method of proof is altered considerably.

Proposition 1. $\rho$ (resp. $\tau$ ) is a mapping of $Y_{1}$ (resp. $X_{1}$ ) onto $X$ (resp. Y).

Proof. Let $x$ be any point of $X$ and let $\left\{U_{i}: i \in I\right\}$ be the family of neighborhoods of $x$, where the set of indices $I$ is directed in the usual manner by set inclusion $\left(i_{1} \leqq i_{2}\right.$ if $\left.U_{i_{2}} \subseteq U_{i_{1}}\right)$. For each index $i$, let $f_{x, i}$ be an element of $C_{0}(X)$ with $f_{x, i}(x)=\left\|f_{x, i}\right\|=1$, and $f_{x, i}\left(x^{\prime}\right)=0$ for all $x^{\prime} \in X-U_{i}$. Then it is clear that

$$
\lim _{i}\left(\phi\left(f_{x, i}\right)\right)(y)=\lim _{i} \int \phi\left(f_{x, i}\right) d \mu_{y}=\lim _{i} \int f_{x, i} d\left(\phi^{*} \mu_{y}\right)
$$

exists for all $y \in Y$, and is equal to $\left(\phi^{*} \mu_{y}\right)(\{x\})$.

We wish to show that there exists at least one $y \in Y$ such that $\lim _{i}\left|\left(\phi\left(f_{x, i}\right)\right)(y)\right|>M$. To this end, for each $i \in I$ we will denote by $S_{i}$ the subset of $Y$ defined by

$$
S_{i}=\left\{y \in Y:\left|\left(\phi\left(f_{x, i}\right)\right)(y)\right|>M\right\} .
$$

(Note that $S_{i}$ is nonvoid since $\phi$ is norm-increasing.) We then denote by $Y_{x}$ the subset of all $y \in Y$ such that there exists a net $\left\{y_{i}: i \in I\right\}$ in $Y$, with $y_{i} \in S_{i}$ for each $i$, which has $y$ as a cluster point.

First of all, we claim that $Y_{x}$ is a finite set. For assume that $y$ is any element of $Y_{x}$ and $g_{y}$ is an element of $C_{0}(Y)$ with $g_{y}(y)=\left\|g_{y}\right\|=M$. Let $V$ be the neighborhood of $y$ defined by $V=\left\{y^{\prime} \in Y:\left|g_{y}\left(y^{\prime}\right)\right|>\|\phi\| / 2\right\}$. For each $i \in I$ such that $S_{i} \cap V$ is nonvoid, we choose $y_{i} \in S_{i} \cap V$ and define the complex number $\lambda_{i}$ by $\left|\lambda_{i}\right|=1$ and $\arg \lambda_{i}=\arg g_{y}\left(y_{i}\right)$ $-\arg \left(\phi\left(f_{x, i}\right)\right)\left(y_{i}\right)$; then we have $\left\|\lambda_{i} \phi\left(f_{x, i}\right)+g_{y}\right\|>M+\|\phi\| / 2$, so that $\left\|\lambda_{i} f_{x, i}+\phi^{-1}\left(g_{y}\right)\right\|>M /\|\phi\|+\frac{1}{2}>1$.

Now $\left\|\phi^{-1}\left(g_{y}\right)\right\| \leqq M<1$, so that the maximum set of the function $\left|\lambda_{i} f_{x, i}+\phi^{-1}\left(g_{y}\right)\right|$ is contained in the neighborhood $W_{i}$ of $x$ defined by $W_{i}=\left\{x^{\prime} \in X: f_{x, i}\left(x^{\prime}\right) \neq 0\right\}$. Moreover, at any point $x^{\prime}$ of this maximum set $\left|\left(\phi^{-1}\left(g_{y}\right)\right)\left(x^{\prime}\right)\right|$ is bounded away from zero by the positive quantity $\epsilon=M /\|\phi\|-\frac{1}{2}$. Since $y \in Y_{x}$, there exists a net $\left\{y_{i}\right\}$ in $Y$, with $y_{i} \in S_{i}$ for each $i$, that has $y$ as a cluster point. And for each $i$ such that $y_{i} \in V$, there exists a point $x_{i}$ of the corresponding set 
$W_{i}$ in $X$ with $\left|\left(\phi^{-1}\left(g_{y}\right)\right)\left(x_{i}\right)\right| \geqq \epsilon$. Since the $W_{i}$ thus obtained constitute a neighborhood basis at $x$, we conclude that $\left|\left(\phi^{-1}\left(g_{y}\right)\right)(x)\right| \geqq \epsilon$.

But this clearly implies that $Y_{x}$ is finite. For given any $m$ points $y_{1}, \cdots, y_{m}$ of $Y_{x}$, we can choose the corresponding functions $g_{y_{k}}$ with disjoint supports, so that for any choice of complex numbers $\gamma_{1}$, $\cdots, \gamma_{m}$, with $\left|\gamma_{k}\right|=1, k=1, \cdots, m$, we have $\left\|\sum_{k=1}^{m} \gamma_{k} g_{y_{k}}\right\|=M$. But if we choose the $\gamma_{k}$ such that the numbers $\gamma_{k}\left(\phi^{-1}\left(g_{y_{k}}\right)\right)(x)$ have equal arguments, we obtain

$$
\left\|\phi^{-1}\left(\sum_{k=1}^{m} \gamma_{k} g_{\nu_{k}}\right)\right\| \geqq\left|\sum_{k=1}^{m} \gamma_{k}\left(\phi^{-1}\left(g_{\nu_{k}}\right)\right)(x)\right| \geqq m \epsilon .
$$

Hence $Y_{x}$ is finite, say $Y_{x}=\left\{y_{1}, y_{2}, \cdots, y_{n}\right\}$, and we write

$$
\phi^{*-1} \mu_{x}=\sum_{k=1}^{n} \beta_{k} \mu_{y_{k}}+\mu,
$$

where the $\beta_{k}$ are complex numbers and $\mu$ is a regular Borel measure on $Y$ with $\mu\left(\left\{y_{k}\right\}\right)=0$ for $k=1, \cdots, n$. (A very simple argument shows that $Y_{x}$ is nonvoid. However, since this fact will be a consequence of what is proven below, for the moment we simply set $\phi^{*-1} \mu_{x}=\mu$, if $Y_{x}$ is void.) We now show that there exists at least one $y_{k} \in Y_{x}$ with $\lim _{i}\left|\left(\phi\left(f_{x, i}\right)\right)\left(y_{k}\right)\right|>M$. For suppose that for all $y_{k} \in Y_{x}$ we had $\lim _{i}\left|\left(\phi\left(f_{x, i}\right)\right)\left(y_{k}\right)\right| \leqq M$. Then, since $Y_{x}$ is finite, there would be an $i_{1} \in I$ such that for all $i \geqq i_{1}$ and all $y_{k} \in Y_{x}$ we would have $\left|\left(\phi\left(f_{x, i}\right)\right)\left(y_{k}\right)\right|<M+(1-M) / 2$. Next, by the regularity of $\mu$, we could find a compact set $K \subseteq Y-Y_{x}$ such that $|\mu|(Y-K)<(1-M) / 4$. $(|\mu|$ denotes the total variation of $\mu$.) Since $K$ is compact and disjoint from $Y_{x}$, there exists an $i_{2} \in I$ such that $i \geqq i_{2}$ implies $\left|\left(\phi\left(f_{x, i}\right)\right)(y)\right| \leqq M$ for all $y \in K$. Hence, if we choose an $i_{0} \in I$ such that $i_{0} \geqq i_{1}$ and $i_{0} \geqq i_{2}$, then for all $i \geqq i_{0}$ we would obtain (noting that $\sum\left|\beta_{k}\right| \leqq 1$ and $\|\mu\| \leqq 1-\sum\left|\beta_{k}\right|$ )

$$
\begin{aligned}
1= & \int f_{x, i} d \mu_{x}=\int \phi\left(f_{x, i}\right) d\left(\phi^{*-1} \mu_{x}\right) \\
= & \sum \beta_{k} \int \phi\left(f_{x, i}\right) d \mu_{y_{k}}+\int_{K} \phi\left(f_{x, i}\right) d \mu+\int_{Y-K} \phi\left(f_{x, i}\right) d \mu \\
< & \left(\sum\left|\beta_{k}\right|\right)[M+(1-M) / 2]+M\left(1-\sum\left|\beta_{k}\right|\right) \\
& +2(1-M) / 4 \\
= & M+\left(1+\sum\left|\beta_{k}\right|\right)(1-M) / 2 \leqq 1,
\end{aligned}
$$

which is absurd. 
Hence we conclude that there is at least one point of $Y_{x}$, which we denote simply as $y$, such that $\lim _{i}\left|\left(\phi\left(f_{x, i}\right)\right)(y)\right|>M$. Writing $\phi^{*} \mu_{y}$ $=\alpha \mu_{x}+\mu$, where $\alpha$ is complex and $\mu(\{x\})=0$, we have

$$
\lim _{i}\left(\phi\left(f_{x, i}\right)\right)(y)=\left(\phi^{*} \mu_{y}\right)(\{x\})=\alpha,
$$

so that $|\alpha|>M, y \in Y_{1}$, and $\rho(y)=x$.

Similarly, if $y$ is any point of $Y$, a net $\left\{g_{y, j}: j \in J\right\}$ of elements of $C_{0}(Y)$, converging pointwise to the characteristic function of $\{y\}$, is defined in a manner exactly analogous to that used in defining the $f_{x, i}$. If we then define the subset $T_{j}$ of $X$ by $T_{j}=\{x \in X$ : $\left.\left|\left(\phi^{-1}\left(g_{y, j}\right)\right)(x)\right|>N\right\}$, and define $X_{y}$ to be the set of all $x \in X$ such that there exists a net $\left\{x_{j}\right\}$ in $X$, with $x_{j} \in T_{j}$ for all $j$, which has $x$ as a cluster point, an argument analogous to that given above shows that

(a) $X_{y}$ is finite, and

(b) for at least one point $x \in X_{y}$, we have $\lim _{j}\left|\left(\phi^{-1}\left(g_{y, j}\right)\right)(x)\right|>N$. (In order to establish (a), the constants $M$ and $\|\phi\| / 2$ which appear in the proof above may be replaced by $N$ and $\frac{1}{2}$, respectively. While to establish (b), the constants analogous to $(1-M) / 2$ and $(1-M) / 4$ may be taken to be $(1-N\|\phi\|) / 2$ and $(1-N\|\phi\|) / 4$, respectively.) Then for the $x$ given by (b), we find that $\phi^{*-1} \mu_{x}=\beta \mu_{y}+\mu, \beta$ complex, $|\beta|>N, \mu(\{y\})=0$, and the statements about $X_{1}$ and $\tau$ follow.

Proposition 2. If $y \in Y_{1}$ and $\rho(y)=x$, then $x \in X_{1}$ and $\tau(x)=y$.

Proof. Let $y$ belong to $Y_{1}$ and let $\left\{g_{y, j}: j \in J\right\}$ be a net of elements of $C_{0}(y)$, converging pointwise to the characteristic function of $\{y\}$, and defined as in the proof of the previous proposition. Let $\rho(y)=x$ and let us assume that either $x$ is not an element of $X_{1}$, or that $x$ belongs to $X_{1}$ but $\tau(x) \neq y$. We must then conclude that $\lim _{j}\left|\left(\phi^{-1}\left(g_{y, j}\right)\right)(x)\right| \leqq N$. We now note that if $x^{\prime}$ is any element of $X$ such that $\lim _{j}\left|\left(\phi^{-1}\left(g_{y, j}\right)\right)\left(x^{\prime}\right)\right|>N$, then $x^{\prime}$ belongs to $X_{y}$, a finite set. Hence if we define the number $P$ by

$$
P=\sup _{x^{\prime} \in X} \lim _{j}\left|\left(\phi^{-1}\left(g_{y, j}\right)\right)\left(x^{\prime}\right)\right|
$$

there exists an $x_{1} \in X_{y}$ with

$$
\lim _{j}\left|\left(\phi^{-1}\left(g_{y, j}\right)\right)\left(x_{1}\right)\right|=P .
$$

Now by assumption $x_{1} \neq x$, so that there exists a $y_{1} \in Y_{1}, y_{1} \neq y$, with $\rho\left(y_{1}\right)=x_{1}$. This means that $\phi^{*} \mu_{y_{1}}=\alpha \mu_{x_{1}}+\mu$, with $|\alpha|>M$ and $\mu\left(\left\{x_{1}\right\}\right)=0$. We now write 


$$
\mu=\sum_{k=2}^{n} \alpha_{k} \mu_{x_{k}}+\nu
$$

where $\left\{x_{1}, x_{2}, \cdots, x_{n}\right\}$ is the set $X_{y}$, and $\nu\left(\left\{x_{k}\right\}\right)=0, k=1,2, \cdots, n$. Note that

$$
\sum_{k=2}^{n}\left|\alpha_{k}\right|+\|\nu\|=\|\mu\|<M^{\prime}
$$

Since $M>M^{\prime}$, we may choose a positive number $\epsilon$ such that $(P-\epsilon) M>(P+\epsilon) M^{\prime}$. We may then find a $j_{1} \in J$ such that for all $j \geqq j_{1}$, we have $\left|\left(\phi^{-1}\left(g_{y, j}\right)\right)\left(x_{1}\right)\right|>P-\epsilon$ and $\left|\left(\phi^{-1}\left(g_{\nu, j}\right)\right)\left(x_{k}\right)\right|<P+\epsilon$, $k=2, \cdots, n$. Next, since $|\nu|\left(X_{y}\right)=0$, we can find a compact set $K \subseteq X-X_{y}$ such that $|\nu|(X-K) \leqq[(P+\epsilon)-N]\|\nu\|$. Because $K$ is compact and disjoint from $X_{y}$, there exists a $j_{2} \in J$ such that if $j \geqq j_{2}$, $\left|\left(\phi^{-1}\left(g_{y, j}\right)\right)\left(x^{\prime}\right)\right| \leqq N$ for all $x^{\prime} \in K$. We then choose $j_{0} \in J$ such that $j_{0} \geqq j_{1}, j_{0} \geqq j_{2}$, and such that for all $j \geqq j_{0}$ the support of $g_{\nu, j}$ does not contain the point $y_{1}$. Hence for $j \geqq j_{0}$, we have

$$
\begin{aligned}
0= & \int g_{y, j} d \mu_{y_{1}}=\int \phi^{-1}\left(g_{y, j}\right) d\left(\phi^{*} \mu_{y_{1}}\right) \\
= & \alpha \int \phi^{-1}\left(g_{y, j}\right) d \mu_{x_{1}}+\sum_{k=2}^{n} \alpha_{k} \int \phi^{-1}\left(g_{y, j}\right) d \mu_{x_{k}} \\
& +\int_{X-K} \phi^{-1}\left(g_{y, j}\right) d \nu+\int_{K} \phi^{-1}\left(g_{\nu, j}\right) d \nu .
\end{aligned}
$$

But for all $j \geqq j_{0}$, the modulus of the first term on the right is greater than $(P-\epsilon) M$, while the modulus of the sum of the remaining terms is less than or equal to

$$
\begin{gathered}
(P+\epsilon)\left(\sum_{k=2}^{n}\left|\alpha_{k}\right|\right)+[(P+\epsilon)-N]\|\nu\|+N\|\nu\| \\
=(P+\epsilon)\|\mu\|<(P+\epsilon) M^{\prime},
\end{gathered}
$$

and this contradiction completes the proof of the proposition.

Proposition 3. $Y_{1}=Y$ and $\rho$ is a homeomorphism of $Y$ onto $X$.

The proof is the same as that given for Proposition 3 of [1].

\section{REFERENCE}

1. M. Cambern, A generalized Banach-Stone theorem, Proc. Amer. Math. Soc. 17 (1966), 396-400.

University of California, Santa Barbara 